\title{
Géolinguistique
}

16 | 2016

Varia

\section{Le parcours de Th. Lalanne : du recueil de données (1947-1952) aux cartes synthétiques de l'Atlas linguistique de la Gascogne maritime}

Th. Lalanne's Work: From Data Collection (1947-1952) to Synthetic Maps of the Atlas linguistique de la Gascogne maritime

\section{Jean-Louis Fossat}

\section{OpenEdition \\ Journals}

Édition électronique

URL : http://journals.openedition.org/geolinguistique/517

DOI : 10.4000/geolinguistique.517

ISSN : 2650-8176

\section{Éditeur}

UGA Éditions/Université Grenoble Alpes

\section{Édition imprimée}

Date de publication : 1 décembre 2016

Pagination : 107-129

ISBN : 978-2-84310-342-1

ISSN : 0761-9081

\section{Référence électronique}

Jean-Louis Fossat, «Le parcours de Th. Lalanne : du recueil de données (1947-1952) aux cartes synthétiques de l'Atlas linguistique de la Gascogne maritime », Géolinguistique [En ligne], 16 | 2016, mis en ligne le 15 février 2019, consulté le 30 octobre 2020. URL : http://journals.openedition.org/ geolinguistique/517 ; DOI : https://doi.org/10.4000/geolinguistique.517 


\title{
Le parcours de Th. Lalanne : du recueil de données (1947-1952) aux cartes synthétiques de l'Atlas linguistique de la Gascogne maritime
}

\author{
Jean-Louis Fossat \\ Université Toulouse 2 - Le Mirail
}

\section{Résumé}

L'Atlas linguistique et ethnographique de la Gascogne (ALG), entreprise du Nouvel Atlas linguistique de la France par régions - initialement sous la direction d'Albert Dauzat - a été élaboré entre 1950 et 1973. Dès les années 1950, Th. Lalanne (l'abbé Lalanne), a été pour le domaine gascon aquitanique le principal enquêteur de l'ALG, pour les volumes 1, 2, 3 d'une enquête reposant sur la méthode directe de transcription sur carnets (49 carnets). Il avait ensuite dressé un petit Atlas linguistique de la Gascogne maritime qui est composé de trois carnets de cartes analytiques et synthétiques ou cumulatives, aujourd'hui numérisé. Th. Lalanne, par le biais d'opérations de décompte élémentaire des oppositions phonétiques, phonologiques, morphologiques, syntaxiques et prosodiques, et après visualisation cartographique du partitionnement de ce territoire, en est arrivé à formuler l'hypothèse, généralisable aux organisations dialectales, d'un ordre par sous-ensembles graduels, c'est-à-dire flous ; ce que, vingt ans plus tard, avant même toute vérification par les méthodes de classification, Jean Séguy systématisa, pour l'ensemble gascon, sous le titre gradient de gasconité dans ALG 6. De là à formuler l'hypothèse d'une organisation en fractales, il n'y a qu'un pas; et ce pas n'est pas d'ordre métaphorique : le travail de vérification qui reste à faire, à partir de là, est immense et fascinant.

\section{Mots-clés}

Bases de données dialectales, ensembles et sous-ensembles graduels, géographie linguistique, gascon, occitan. 


\begin{abstract}
From Lalanne-1950 to Séguy-1973 (ALG 6 Gasconish Dialectometry), there is a long way. From Lalanne Maritim Gasconish dialectal data base to Linguistic Gradual Sets and Fuzzy Subsets (ALG 6 phonetic data; phonological data; morphosyntactic data; lexical data Fuzzy Sets): we have now achived to validate the first hypotesis on dialectal organization by Fuzzy Sets and Subsets. And now? what does say us about Dialectal Organization by "Ward's Method" of Distance Calculation? and how calculate that we name "Dialectal Fractals"? That is the crucial question.
\end{abstract}

\title{
Keywords
}

Dialectal databases, gradual units and subsets, Linguistic geography, Gascon, Langue d'oc.

La constitution d'une base de données des carnets et cartes de la Gascogne maritime, œuvre de l'abbé Lalanne ne répond pas à une obligation de fidélité, et pas davantage à un goût de l'accumulation de données dialectales empilées hors analyse.

L'objectif visé ici est de retracer le parcours de l'abbé Lalanne (19471950), de Saint-Vincent-de-Paul (Landes), dans son analyse du partitionnement flou des données dialectales concernant la Gascogne maritime, et de souligner son intérêt majeur du point de vue de l'évolution des méthodes de traitement du fait dialectal dans l'espace et dans le temps des sociétés historiques.

Certes, ces données ne concernent que le quadrilatère de la gasconité occidentale de la pointe du Haut-Médoc à Bayonne d'une part, de La Sauve (Entre-deux-Mers) à Parleboscq (Landes) d'autre part. Elles concernent non l'archéologie de l'écrit gascon, mais le parler réel tel que capté par transcription directe, hors enregistrement, dans les années 1950, dans son projet d'analyse de la dynamique des aires dialectales de la Gascogne maritime (Landes de Gascogne). Ce que l'on voudrait souligner ici c'est l'intérêt majeur de l'analyse géolinguistique de Th. Lalanne, qui fonde dès 1950 ce que Jean Séguy dans les années 1970 appellera l'analyse des gradients de gasconité.

Tous les niveaux de description du fait linguistique dialectal peuvent être considérés soit comme autonomes, soit comme reliés en analyse multivariée, selon les degrés de complexité de la covariance de fait observée et analysée, fondatrice de sous-groupes, de sous-ensembles, au sein même du territoire délimité : 
- sous-ensembles géolinguistiques fondés sur l'analyse phonétique diachronique;

- sous-ensembles géolinguistiques fondés sur l'analyse phonologique;

- sous-ensembles géolinguistiques fondés sur l'analyse morphologique et syntaxique ;

- sous-ensembles géolinguistiques fondés sur l'analyse prosodique.

La seule chose cruciale que ne pouvait faire en 1950 Lalanne c'est constituer des ressources numériques en vue de l'analyse de corpus de parole : Anar tot dret : vè-t'en tot dretlanatz tot dret! Autrement dit, aux carnets Lalanne comme aux carnets de l'ALG 1/2/3 il ne manquait que la parole.

\section{Hiérarchisation des entrées selon des critères de complexité}

Th. Lalanne a déterminé trois niveaux de complexité.

\subsection{Complexité minimale MOT dialectal : Ia haie}

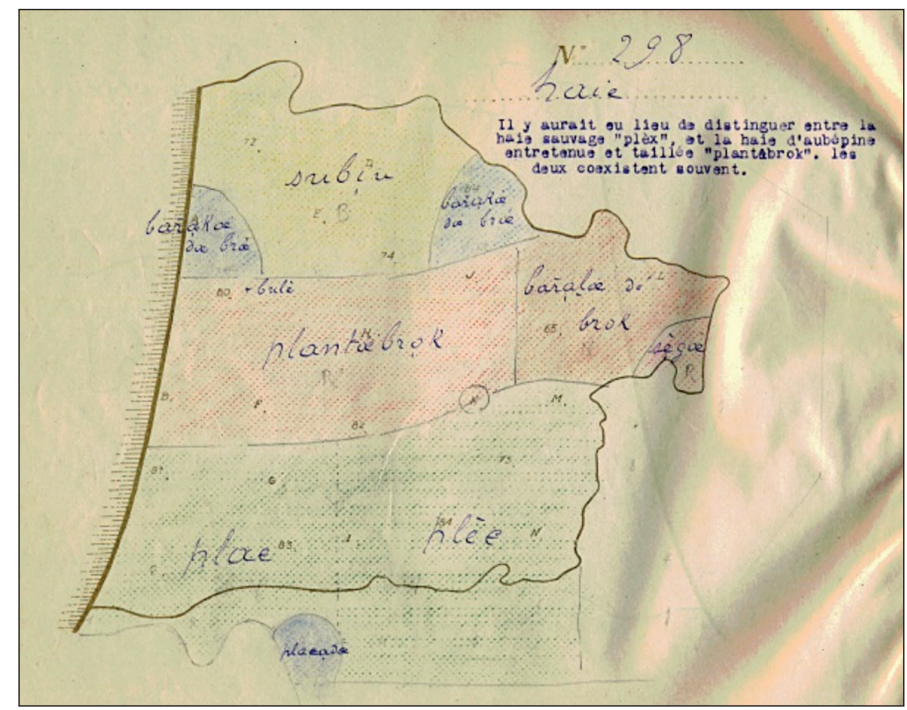

Figure 1. - Carte analytique : 197 haie.

On affiche alors dans la base de données des cartes analytiques les variables positionnées sur le mot ou les syntagmes (1/2207 blocs descriptifs) : (1) variables phonétiques et phonologiques; (2) variables morphosyntaxiques; (3) variables lexicales. Dès 1950 la visualisation par gradient 
de couleurs est proposée par Lalanne, mais non suivie par la stratégie éditoriale du NALF (Nouvel Atlas linguistique de France), pour des raisons aisées à justifier.

\subsection{Complexité moyenne : le syntagme dialectal}

Item $1:$ en rentrant de l'école...

Item $2:$ huit poules... (tête de syntagme) ... [pour un coq]

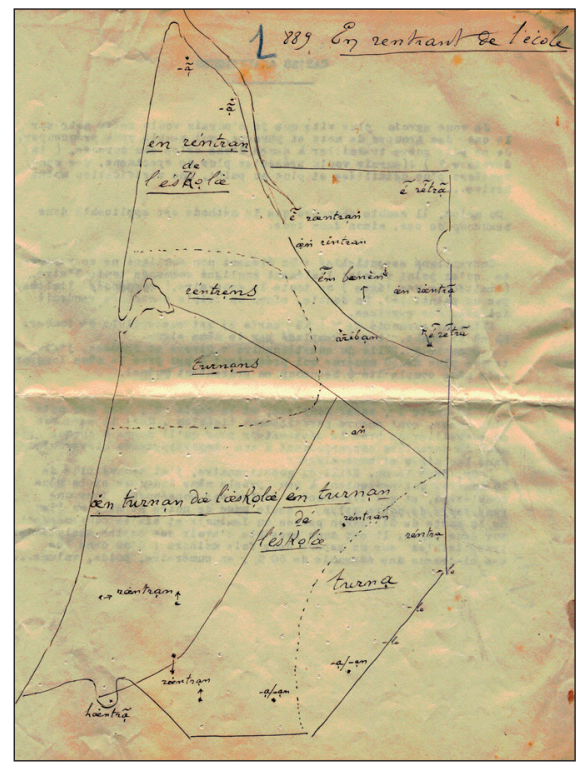

Figure 1 bis.

Carte «en rentrant de l'école».

Figure 2.

Carte «huit poules».

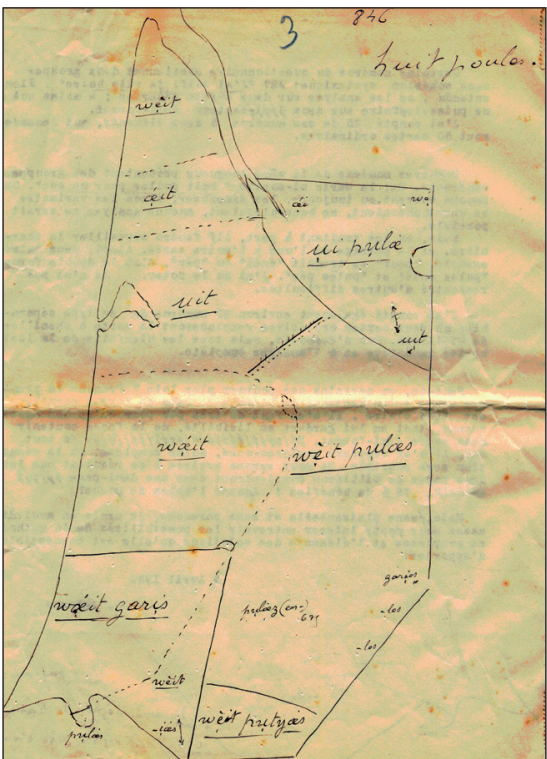

Les cartes des figures 1,1 bis, 2, 2 bis et 3 sont des maquettes prééditoriale. 


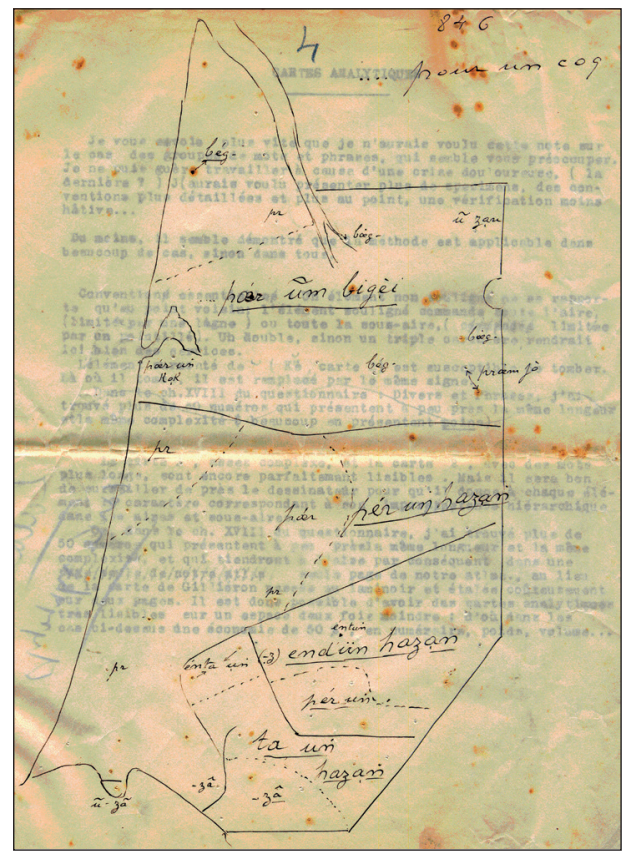

Figure 2 bis. - Carte «pour un coq».

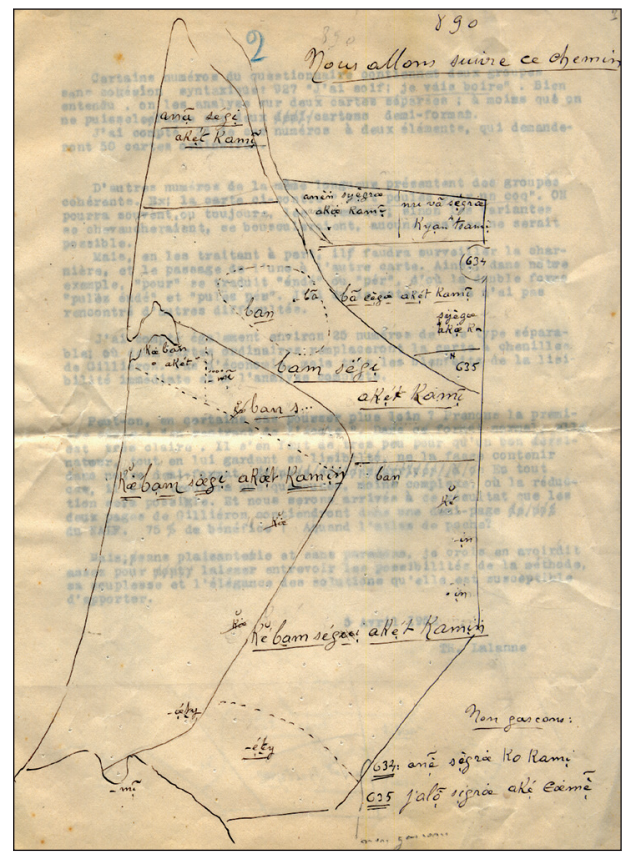

Figure 3. - Carte «nous allons suivre ce chemin ». 
On affiche alors dans la base de données les variables positionnées sur les suites :

(1) variables phonétiques et phonologiques: marqueurs de nasalité marqueurs de labialité

(2) variables morphosyntaxiques : IFCoP/gérondif $\mathrm{CoP}$

\subsection{Complexité maximale : la phrase dialectale}

\begin{tabular}{|c|c|c|c|}
\hline 1689 & $\begin{array}{l}\text { AQUITAINE } \\
\text { MEDOC } \\
\text { GASCOGNE_MARITIME } \\
\text { CHALOSSE } \\
\text { MARENSIN } \\
\text { BORN } \\
\text { BEARN } \\
\text { GERS } \\
\text { GROUPE1 GR_LANDE } \\
\text { LA_TESTE } \\
\text { SALLES/HOSTENS } \\
\text { ST_SYMPHORIEN } \\
\text { CAPTIEUX } \\
\text { HOUEILLES } \\
\text { GROUPE2 BEARN } \\
\text { BIARRITZ } \\
\text { URT } \\
\text { SALIES_BEARN } \\
\text { SAUVETERRE } \\
\text { STE_SUZANNE } \\
\text { CABIDOS } \\
\text { GARLIN } \\
\text { Groupe3 GERS } \\
\text { EAUZE } \\
\text { NOGARO } \\
\text { RISCLE }\end{array}$ & $\begin{array}{l}1950- \\
1952\end{array}$ & $\begin{array}{l}\text { Q_943 oublier/devoir : avez-vous oublié que vous me devez de l'argent? } \\
\text { PREDIC INTERROGATION } \\
\text { partic_interrogatif/se/e/0/1 } \\
\text { PC3_oblidar/desoblidar/desobligar AUX_aver/aver oblidat } \\
\text { PC3_desbrembar/desbrombar-se } \\
\text { VOIX_pronominale/desbrembar-se } \\
\text { que/conjonctif } \\
\text { IP5_faible/dever/devetz } \\
\text { IP5_fort/dever/dévetz/'diwat/'dewət/ } \\
\text { IMPT5_dever/devevetz/devevatz } \\
\text { coefficient_labialité/vélarité/B/W/dever } \\
\text { coefficient_antériorisation/i/devetz/devevatz/devevetz } \\
\text { coefficient_patalité/RJ/R3/argent } \\
\text { coefficient_nasalité/voc/coda/argent/d'argent } \\
\text { PrCo_Datif/me/te/nos/vos/CONTROL_destinataire } \\
\text { PrCo_emphatique/ves/p/ } \\
\text { clise/asyllabique/ves /p/ hôte_clise/AUX/p_ei// } \\
\text { Pr4Sujet/0/1/vos/ves/ } \\
\text { hôte_clise/me/conjonction/que/que'm } \\
\text { actualisant_0/1/de/d'/d'argent/argent/dar argent } \\
\text { N_quant/argent/moneda/sòus } \\
\text { @atz oblidat que me devetz argent/moneda?@ } \\
\text { @qu'avetz/qu'atz oblidat que devetz argent!?@ } \\
\text { @e p'avetz desbrembat que devetz dar argent/moneda?@ } \\
\text { @ e se p'ei desmombrat/desobligat que'm devevatz sòus?@ /de'ßewot/ } \\
\text { @ vos atz desobligat que'm devèvetz/devetz dar argent!?@ }\end{array}$ \\
\hline
\end{tabular}

Bloc descriptif 1659 Q_943 carnets Lalanne (1950)

"vous avez oublié/avez-vous oublié que vous melluil/leur/nous devez de l'argent/ non, je ne l'ai pas oublié».

Ce type de carte lexicale analytique, à partir d'une indexation hiérarchique, répond à la détermination de la cohérence distributionnelle des sous-ensembles lexicaux flous classés selon leur degré de complexité. Pour ce qui est de la détermination lexicographique et la stabilité de la morphologie lexicale nous renvoyons au lexique-grammaire du gascon en ligne aux rubriques de lexique-grammaire formant clés de requête ${ }^{1}$.

1. Pour une analyse du lexique-grammaire sous l'aspect géolinguistique, je renvoie au répertoire Dictionnaires/lexique-grammaire du site <occiton.free.fr >. 
Pour ce qui est de la déclaration des variables, nous renvoyons à présent depuis ALG 6 aux tables de calcul des similarités et distances (Séguy, 1973), traitées en mode d'analyse exploratoire des données, pour la cartographie des classes, groupes, sous-groupes, sous-ensembles.

\subsection{Découverte du principe de partitionnement en sous-ensembles flous (Lalanne, 1950)}

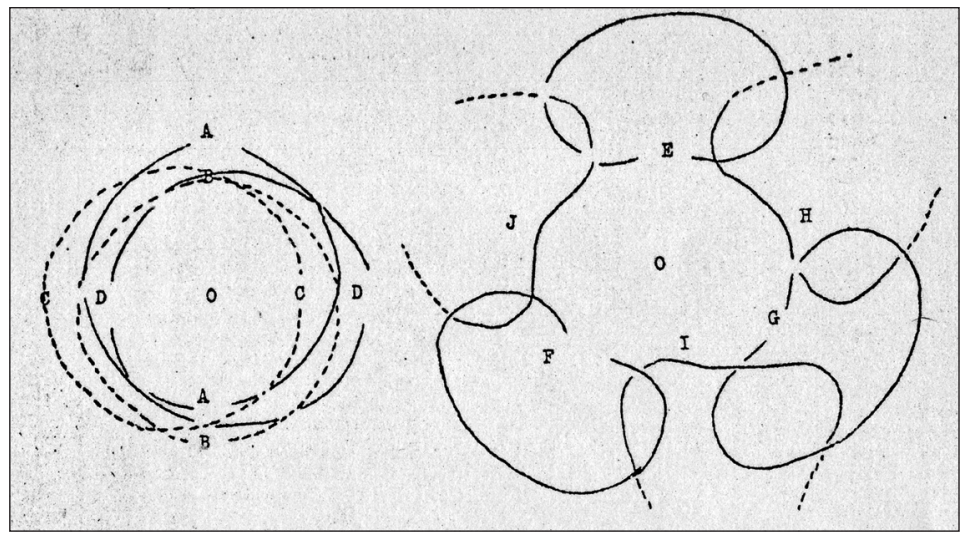

Figure 4. - Représentation par Lalanne du partitionnement territorial organisé en sous-ensembles flous (Lalanne, 1950, 2 : 93).

Dès 1950 Th. Lalanne, dit l'abbé Lalanne, à partir d'un travail précis de cartographie analytique et synthétique, était un des premiers à dénoncer, à partir de leur tracé, le leurre des isoglosses et des frontières dialectales géométriques droites, fussent-elles calculées en distances euclidiennes. Mais personne à cette date, en sciences humaines, ne disposait des outils d'analyse exploratoire permettant des tracés de classes hiérarchiques, fermées ou ouvertes. Toutefois, dans sa thèse sur l'indépendance des aires en Gascogne maritime (Lalanne, 1950), il mettait en évidence l'existence de ce qu'on appellerait aujourd'hui sous-ensembles flous, autrement dit graduels dans des petits mondes fractals, que tous les géolinguistes appellent parlers dialectaux. Et, fort heureusement, l'entreprise du NALF des années 1950 à nos jours, avec P. Gardette, G. Tuaillon, J.-B. Martin, J. Séguy, X. Ravier, J. Allières, L. Michel et J.-M. Petit, J. Boisgontier et J.-C. Bouvier, a persévéré dans sa tâche d'entassement des cartes analytiques avec tracé dynamique, à partir desquelles est rendue possible une cartographie synthétique, cumulative, de classes définies en termes de dynamique de systèmes géolinguistiques complexes (Philps, 1985 ; Fossat et al., 2016). 


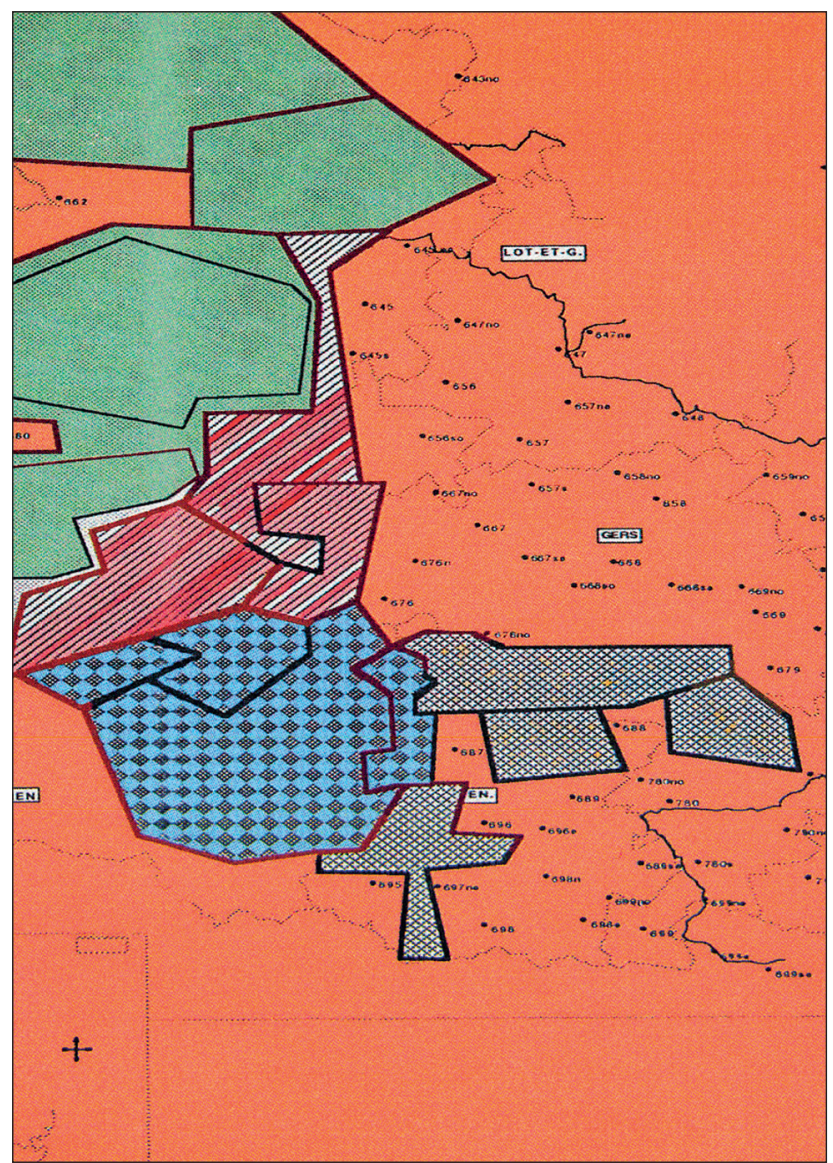

Figure 5. - Carte de classes de phonétique diachronique de la Gascogne maritime (Fossat \& Sandouk, 1991).

De tous les paramètres linguistiques, seul l'arbre des parlers selon les critères de phonétique diachronique présente ce type de morcellement maximal en sous-ensembles historiques (pays) et sociaux; les paramètres structuraux (phonologie et morphosyntaxe présentent un partitionnement minimal qui oppose gascon occidental, central et oriental.

On notera que ce type de représentation par juxtaposition de droites séparatrices de sous-ensembles typologiques (Fossat \& Sandouk, 1978) est juste, à condition d'ouvrir des portes d'interaction entre tout groupe et tout sous-groupe; ce qui revient à réintroduire du flou entre classes communicantes et sécantes; dans sa modélisation de la variation floue Lalanne (1950) avait tracé une représentation géométrique de tels objets fractals. 


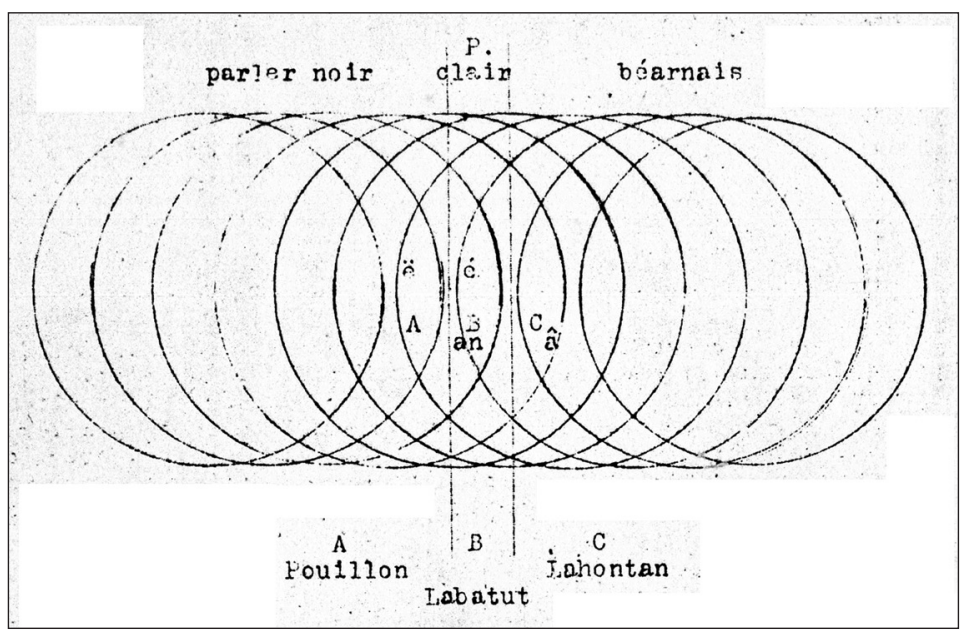

Figure 6. - Sous-ensembles sécants (Lalanne, 1950 : 2, fasc. 1, conclusions II).

C'est ce type de représentation modélisée (Lalanne, 1950) qui permit à Jean Séguy, des années 1950 jusqu'aux années 1970, de mettre en place, manuellement, un dispositif dialectométrique qui permet à tout un chacun de calculer, vérifier, tracer. Ce qui permet, à partir d'un travail organisé, de se faire une idée de ce type d'organisation, universel, des mondes dialectaux, qu'ils soient romans ou non.

\section{Description de la base de données «Lalanne»}

\subsection{Les carnets d'enquête "Lalanne » de la phase NALF ALG 1/2/3}

Le recueil de données premières scannées pour constituer la base de données Gascogne maritime se compose de 49 cahiers d'enquête portant sur 1000 questions (données lexicales, morphonologiques, prosodiques et syntaxiques) et 3 carnets de cartes analytiques et synthétiques qui constituent le prototypage de la cartographie affinée par Jean Séguy dans son Atlas linguistique et ethnographique de la Gascogne, du volume 1 au volume 6. Le questionnaire est celui du NALF/ALG 1/2/3, dit Questionnaire Dauzat. Th. Lalanne fut en effet, à partir de là, l'enquêteur principal de l'ALG 1/ 2/3 sous la direction de Jean Séguy. La base de données que nous avons constituée comporte actuellement 2107 blocs descriptifs, extensibles à 3000 pour ce qui est des carnets, et à 197/300 blocs descriptifs, pour ce qui est des maquettes de représentations cartographiques. On trouvera ci-dessous le bloc descriptif correspondant au jeu des données de la question 943, pour le bloc territorial 01 de Biscarrosse (40) à Parleboscq (40). 
Série $01: 5$ carnets (Q001-1000)

Série 02 : 9 carnets (Q001-1000)

Série 03 : 10 carnets (Q001-1000)

Série 04 : (4 carnets (Q001-0400)

Série 05 : compléments d'enquête, notes, rectificatifs, correspondance

Taille de la base en son état actuel : 2207 blocs d'information synoptique/texte-image sur le site <occiton.free.fr> pour 197 cartes.

Toute image-carte, comme toute image de données/carnet est appariée à une zone texte analytique : ceci constitue la déclaration des variables pour traitement par cartographie analytique/synthétique. Th. Lalanne dans ses carnets d'enquête avait déjà prévisualisé l'existence d'un partitionnement en sous-groupes, que les analyses ultérieures pouvaient confirmer ou infirmer : il avait tracé une ligne droite séparatrice de trois sous-ensembles flous, septentrional, méridional et central. C'est dire que l'objectif était de valider l'hypothèse d'une dynamique des aires dialectales que la méthode des isoglosses singulières ne permettait pas, à elle seule, de mettre en évidence. C'est une approche objet-fractal orientée vers les lecteurs cartographes, qui se représentent le partitionnement territorial à partir de la lecture des feuilles de carnets ainsi partitionnées.

\subsection{Méthodes de cartographie analytique et synthétique, lexique- grammaire}

Comment l'enquêteur Th. Lalanne dans les années 1950 en est-il parvenu à concevoir diverses améliorations aux cartes de l'ALF de Gilliéron et Edmont, pour passer des cartes analytiques du lexique aux cartes analytiques et synthétiques de morphosyntaxe? Ce qu'il formule dans sa correspondance à J. Séguy, est déjà la mise en place d'une conception des sous-ensembles gradients, appelés depuis 1965 par les mathématiciens sousensembles flous, et que j'appellerai, après Lalanne, gradients de complexité/ gradients de fragmentation, conçus en termes mathématiques d'objets fractals. Je laisserai parler Th. Lalanne (correspondance à Jean Séguy datée du 3 avril 1952) :

Je vous envoie plus vite que je n'aurais voulu cette note sur le cas des groupes de mots et phrases, qui semble vous préoccuper... j'aurais voulu présenter plus de spécimens, des conventions plus détaillées et plus au point, une vérification moins hâtive. Du moins, il semble que la «méthode est applicable dans beaucoup de cas, sinon dans tous $»^{2}$.

2. La fouille systématique de la base de données établie à partir des cartes et carnets de l'abbé Lalanne permet d'établir ce qu'il proposait dans les années 1950, une anno- 
Conventions essentielles : un élément non souligné ne se rapporte qu'au point voisin; l'élément souligné commande toute l'aire (limitée par une ligne) ou toute sous-aire, limitée par un pointillé. Un double, sinon un triple caractère rendrait bien des services.

L'élément surmonté de [`], dont la forme graphique est «que» et dont la transcription est $/ \mathrm{ke}^{\curlyvee} / \mathrm{k}^{\curlyvee} /$ (voir carte 3 ), est susceptible de tomber. Là où il tombe, il est remplacé par le même signe ( $($ ). S'ensuit une note de bas de page qui explicite le phénomène. La carte 1 bis, assez complexe, et les cartes 2 et 3, avec des mots plus longs, sont encore parfaitement lisibles. Mais il sera bon de surveiller de près le dessinateur pour qu'il donne à chaque élément le caractère correspondant à son importance hiérarchique dans les aires et sous-aires.

Or, dans le chapitre XVIII du questionnaire, j'ai trouvé plus de 50 numéros qui présentent à peu près la même longueur et la même complexité, et qui tiendront à l'aise, par conséquent dans une seule page de notre atlas, au lieu de la carte de Gilliéron passée au laminoir et étalée coûteusement sur deux pages. Il est donc possible d'avoir des cartes analytiques très lisibles sur un espace deux fois moindre; d'où, dans les cas ci-dessus une économie de $50 \%$, en numéraire, poids, volume...

Certains numéros du questionnaire contiennent deux groupes sans cohésion syntaxique : 927 j'ai soif; je vais boire. Bien entendu, on les analyse sur deux cartes séparées; à moins qu'on ne puisse le faire sur deux cartons demi-format. D'autres numéros de même longueur présentent des groupes cohérents; p. ex. la carte ci-dessus huit poules pour un coq; on pourra toujours ou souvent les démembrer, «sinon les variantes se chevaucheraient; se bousculeraient, aucune analyse ne serait possible».

tation systématique de la trace des mesures de durée entraînant, à débit rapide, des mouvements phonétiques qui ont pour effet une modification des structures phonologiques; qu'il s'agisse d'amuïssement par élision ou par aphérèse, d'extramétricité vocalique de voyelles latentes, d'extramétricité consonantique de consonnes latentes ou des mouvements d'anaptyx et contre-anaptyx affectant les groupes consonantiques combinés $/ \mathrm{BL} / \mathrm{BR} / \mathrm{KR} / \mathrm{GR} /$. Ces mouvements de réduction, annotés, affectent ainsi, entre autres, toutes les suites de que_énonciatif ou du représentant_neutre $(a)$ quò dans tous les contextes possibles :
(a)quò_es eu /ko_'z ew/ c'est lui
(a)quo_ei tu, /ko_j 'ty/ c'est toi

Ces faits sont particulièrement importants pour la détermination des caractéristiques prosodiques des sous-ensembles interférentiels localisés aux marches du domaine gascon et du domaine languedocien, explorées autant par les enquêtes de l'Atlas de la Gascogne (ALG 123) que par l'Atlas linguistique du Poitou et l'Atlas linguistique du Languedoc occidental (enquêtes de E. Négre, X. Ravier et J. Boisgontier). 
Mais, en les traitant à part, il faudra surveiller la «charnière, et le passage de l'une à l'autre carte». Ainsi, dans notre exemple POUR se traduit par ende ou per; d'où la double forme ['puləz ende et 'puləs per].

J'ai compté également 25 numéros de ce type séparables où deux cartes ordinaires remplaceront la carte à chenilles de Gilliéron. Pas d'économie, mais tous les bienfaits de la «lisibilité immédiate et de l'analyse complète».

Peut-on, en certains cas, aller plus loin?

Prenons la carte 1 bis en rentrant de l'école: dans ce format normal elle est très claire. Il s'en faut de très peu pour qu'un bon dessinateur, tout en lui gardant sa lisibilité, ne la fasse contenir dans notre demi-format. En tout cas, il s'en trouvera quelques-unes, moins complexes, où la réduction sera possible. Et nous serons arrivés à ce résultat que les deux pages de Gilliéron contiendront dans une demi-page du NALF : $75 \%$ de bénéfice!

À quand l'atlas de poche? Mais, sans plaisanterie et sans paradoxe, je crois en avoir dit assez pour laisser entrevoir les possibilités de la méthode, sa souplesse et l'élégance des solutions qu'elle est susceptible d'apporter.

Suivent alors les brouillons de spécimens de visualisation du partitionnement qui ont été présentés ici en $\mathbf{1}$ :

carte 1 bis : 889 en rentrant de l'école;

cartes 2 et 2 bis : 846a huit poules... 846b pour un coq;

carte 3 : 890 nous allons suivre ce chemin.

\subsection{Méthodes de représentation géolinguistique des relations de covariance sur énoncé complexe : premières approches de représentation de la variabilité à niveau phrastique}

On partira d'un énoncé type : avez-vous oublié que vous deviez de l'argent? (fig. 7)

Le problème posé par Lalanne/J. Séguy dans les années 1950 est celui de la représentation cartographique analytique et synthétique des entrées syntaxiques : ainsi avez-vous oublié que vous m'en devez? que vous lui en devez? que vous leur en devez tant. 


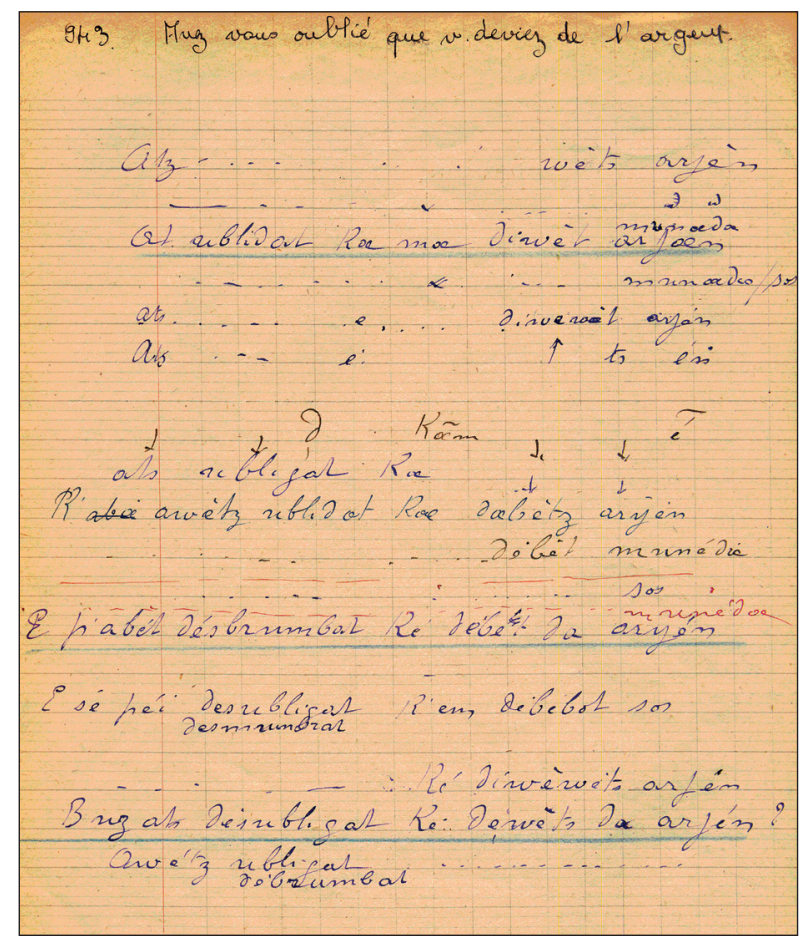

Figure 7. - Carnets Lalanne (1950) «avez-vous oublié que vous deviez de l'argent?»

La déclaration de variables structurales met en évidence les relations de covariance entre les 12 variables suivantes, pour le moins :

\subsubsection{Variables de morphosyntaxe}

option_interrogation/e/se/0/1

option PRSujet/01

option/PC/PT

option IPfort/faible/dever

option_IPaux/réduction/0/1

option actualisant_partitif/0/1

option/IP/IMPT

option_clise/que_me hôte_clise/que

\subsubsection{Phonologie}

option_labialisation/0/1

option/grad/palatalité/RJ/R3/argent

option_coda/NT/réduction

option_labialisation/vélarisation/B/W/dever 


\section{La partition territoriale et la dynamique de la fragmentation en aires et sous-aires dialectales}

La partition territoriale de départ repose sur une hypothèse de la fragmentation des aires en sous-aires hiérarchisées, discriminées par un gradient de tracé.

\subsection{Groupe 1 : Landes}

Ce groupe se laisse décomposer en 6 blocs ou sous-ensembles territoriaux de départ.

\subsubsection{Sous-ensemble 1 \\ 01 Biscarrosse \\ 02 Parentis \\ 03 Mimizan \\ 04 Moustey}

\subsubsection{Sous-ensemble 2}

05 Labouheyre

06 Sabres

07 Luxey

08 Labrit

\subsubsection{Sous-ensemble 3}

09 Ygos

10 Mézos

11 Vielle

\subsubsection{Sous-ensemble 4}

12 Castets

13 Tartas

14 St-Vincent-de-Paul

15 St-Vincent-de-Tirosse

16 Soustons

\subsubsection{Sous-ensemble 5}

17 Pouillon

18 Pomarez

19 Hagetmau

20 Geaune

21 Grenade 


\subsubsection{Sous-ensemble 6}

22 Mazerolle

23 Villleneuve

24 Sarbazan

25 Lubbon

26 Parleboscq

On examinera ici, à partir des carnets, le mode de représentation du lexique-grammaire des actualisants; un òme; ua hemna; la hèmna; las hemnas, avant de se référer aux cartes distributionnelles de l'ALG (Séguy, 1971, ALG 6, cartes distributionnelles, tables).

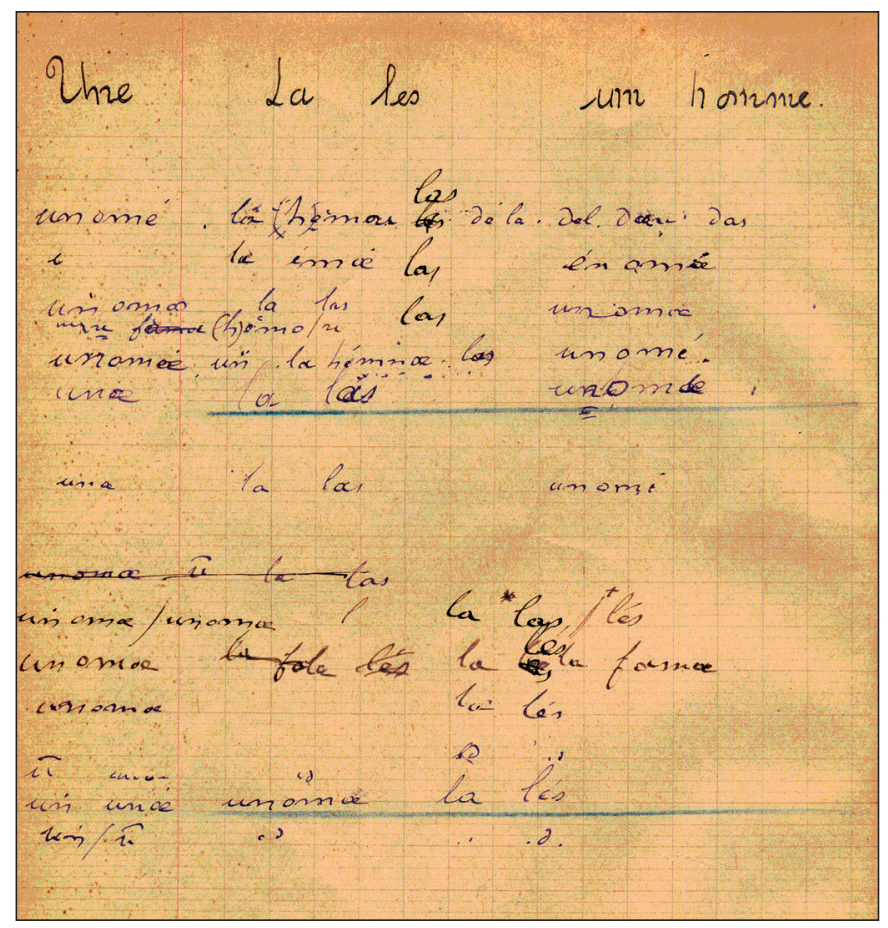

Figure 8. - Carnets Lalanne (1950) ua hemna.

\subsection{Groupe 2 : Gironde}

Ce groupe se laisse décomposer en trois sous-ensembles territoriaux de départ.

\subsubsection{Sous-ensemble 1}

01 Puynormand 634NO ALG 


\subsubsection{Sous-ensemble 2}

02 St-André-de-Cubzac 630S ALG

03 Grézillac 643NE ALG

04 Beychac 643NO ALG

05 La Sauve 6430 ALG

06 Blasimon 643E ALG

07 Vélines 634 ALG

\subsubsection{Sous-ensemble 3}

08 St-Vivien 635 ALG

09 St-Ferme 635NO ALG

10 Abzac

\subsection{Groupe 3 : pourtours groupe interférentiel}

\subsubsection{Sous-ensemble 1}

08 St-Vivien

11 St-Yzans

12 Cissac

13 Hourtin

14 Lacanau

\subsubsection{Sous-ensemble 2}

\section{Castelnau}

\subsubsection{Sous-ensemble 3}

16 St-Jean-d'Illac

17 Pessac

18 Saucats

19 Pujols

20 St-Côme

21 Blaignac

Ce type de zone interférentielle (fig. suiv.), amphizone des géolinguistes (P. Gardette, G. Tuaillon et al.) est essentiel pour cerner les hypothèses de Lalanne (Lalanne, 1950) sur le gradient de stabilité des aires phonétiques, morphologiques, depuis validées par Jean Séguy, sur le leurre des frontières dialectales tracées par isoglosses ou bourrelets d'isoglosses covariantes.

Au total l'enquête de Lalanne se laisse ramener à $36+11=47$ localités gasconnes indexées représentant les parlers de la Gascogne maritime et de ses pourtours ou confins interférentiels. 


\begin{tabular}{|c|c|c|c|}
\hline 0159 & $\begin{array}{l}\text { AQUITAINE } \\
\text { MEDOC } \\
\text { GASCOGNE_MARITIME } \\
\text { CHALOSSE } \\
\text { MARENSIN } \\
\text { BORN } \\
\text { ALG123_LEXIQUE } \\
\text { zone1 } \\
\text { St-Vivien } 548 \\
\text { St-Yzans } \\
\text { Cissan } 439 \\
\text { Hourtin } \\
\text { Lacanau } 650 \\
\text { Castelnau } \\
\text { zone2 } \\
\text { St-Jean-d'Illac } \\
\text { Pessac } \\
\text { Saucats } \\
\text { Pujols } \\
\text { St-Côme } 645 \\
\text { Blaignac }\end{array}$ & $\begin{array}{l}1949- \\
1952\end{array}$ & $\begin{array}{l}\text { Q_000a une femme } \\
\text { ua hemna } \\
\text { SFSG_u/hemna } \\
\text { coefficient_postériorisation/u/hema/'hemu/'hemo/ } \\
\text { SFPLUR_las/les/art_défini } \\
\text { coefficient_centralisation/a/ } \\
\text { coefficient_antériorisation/las/les } \\
\text { coefficient_/F/H/0/distribution } \\
\text { coefficient_assimilation/MB/NN/M/ } \\
\text { Q_000b un homme } \\
\text { un òme } \\
\text { SMSG_e/u } \\
\text { coefficient_Vaton/e/schwa } \\
\text { coefficient_liaison/nasalité/N/vélarité/un òme } \\
\text { del/deu/art_contracte } \\
\text { coefficient_centralisation/a/deu/dau/art_défini } \\
\text { dau/daus/das/art_contracte/PLUR } \\
\text { coefficient_centralisation/aw/a } \\
\text { Q_00c le/las/les/des/de la } \\
\text { coefficient_e/y/un òme/art_défini } \\
\text { SFPLUR_las/les/art_défini } \\
\text { coefficient_centralisation/a/ } \\
\text { coefficient_antériorisation/las/les } \\
\text { coefficient_centralisation/a/deu/dau/art_défini } \\
\text { dau/daus/das/art_contracte/PLUR } \\
\text { coefficient_centralisation/aw/a }\end{array}$ \\
\hline
\end{tabular}

Base de données Lalanne (1950) ua hemna Groupe 3 interférentiel.

On observera attentivement la partition territoriale de départ, inventée par Lalanne (Lalanne, 1950) qui a abouti dès 1950 à une visualisation cartographique par graphes coloriés. Ce mode de visualisation vaut instruction de travail pour l'étape cartographique analytique autant que pour l'étape ultérieure de cartographie synthétique.

\section{L'indexation analytique de la base de données Lalanne (1950)}

L'objectif de l'indexation est de faciliter aux usages leurs requêtes par variables, autrement dit par critères de variabilité, comme le montre le tableau ci-dessus, afin de dresser, à partir de la cartographie analytique de départ, une représentation cartographique de synthèse par variables, avec l'outillage et les méthodes dont pouvait disposer la recherche au milieu du $\mathrm{XX}^{\mathrm{e}}$ siècle.

\subsection{Oppositions phonétiques/phonologiques/prosodiques}

coefficient_palatalité

coefficient_antériorisation

clise 


\subsection{Oppositions morphologiques}

PC3_oblidar/desoblidar/desobligar AUX_aver/aver oblidat

PC3_desbrembar/desbrombar-se

VOIX_pronominale/desbrembar-se

que/conjonctif

IP5_faible/dever/devetz

IP5_fort/dever/dévetz /'diwət/'dewət/

IMPT5_dever/devevetz/devevatz

\subsection{Oppositions syntaxiques}

PrCo_Datif/me/te/nos/vos/CONTROL_destinataire

PrCo_emphatique/ves /p/

clise/asyllabique/ves /p/ hôte_clise/AUX/p_ei/

Pr4Sujet/0/1/vos/ves/

hôte_clise/me/conjonction/que/que'm

actualisant_0/1/de/d'/d'argent/argent/dar argent

À ce stade, les clés de requête analytique indexées facilitent l'étape de fouille de données dans la base, pour des utilisateurs soucieux de repérer les variables caractéristiques de la gasconité dialectale dans les divers sousensembles territoriaux. On reviendra sur ce point en présentant les cartes analytiques et la cartographie synthétique.

\section{Cartes cumulatives synthétiques}

À chaque image de carte analytique/synthétique est associée une analyse par clés de requête. Les cartes synthétiques de Lalanne sont l'ancêtre, le prédécesseur inoubliable des cartes synthétiques distributionnelles de l'ALG que nous avons éditées à partir des tables de variables établies par Jean Séguy pour la totalité des 153 localités de l'ALG. On consultera trois volumes de ces cartes distributionnelles sur notre site <occiton.free.fr>. Nous en donnons ici quelques exemples significatifs.

\subsection{Gradient de variabilité du système vocalique}

Sous-ensemble-flou : gradient/labialité/palatalité/antériorité/Vers la timbre labial/palat /y/ 


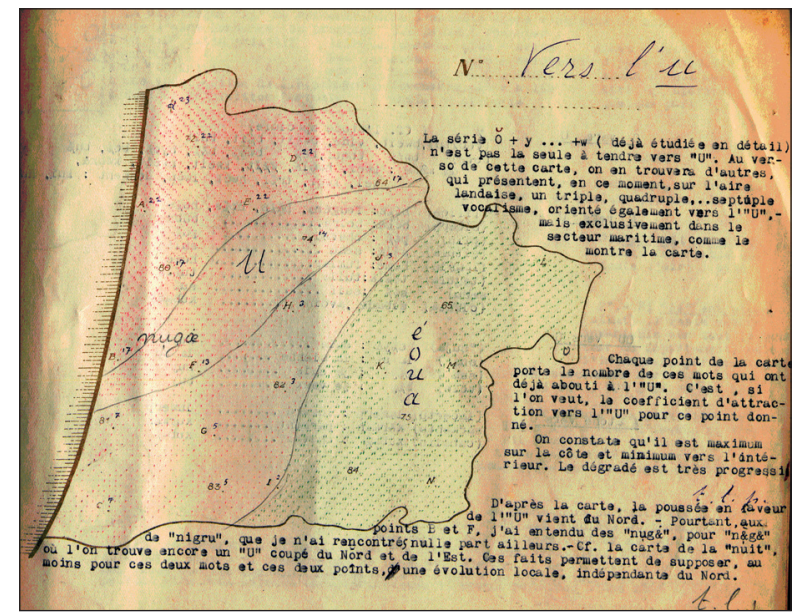

Figure 9. - Carte cumulative synthétique gradient de palatalité/labialité.

\subsection{Complexité non résolue}

On s'attardera à présent plus spécialement sur les cartes synthétiques déclarées complexes, sinon impossibles par Lalanne, dans une perspective ouvrière de fabrique de visualisation des gradients de complexité explicables en termes de covariance.

Pour ce faire, on examinera les prototypes de cartes distributionnelles que construit Lalanne (1950) pour identifier le sous-système flou des variables phonétiques de diphtongaison conditionnée des voyelles toniques ouvertes du gascon (occitan occidental).

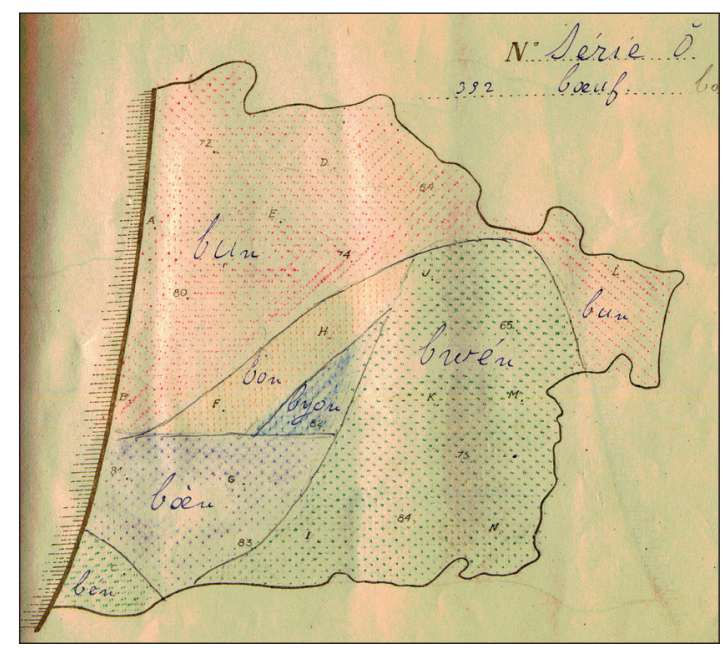

Figure 10. - Carte synthétique gradient de diphtongaison/labialité/palatalité. 


\subsection{Variable de flux concernant le statut de la diphtongue centralisée /aw/}

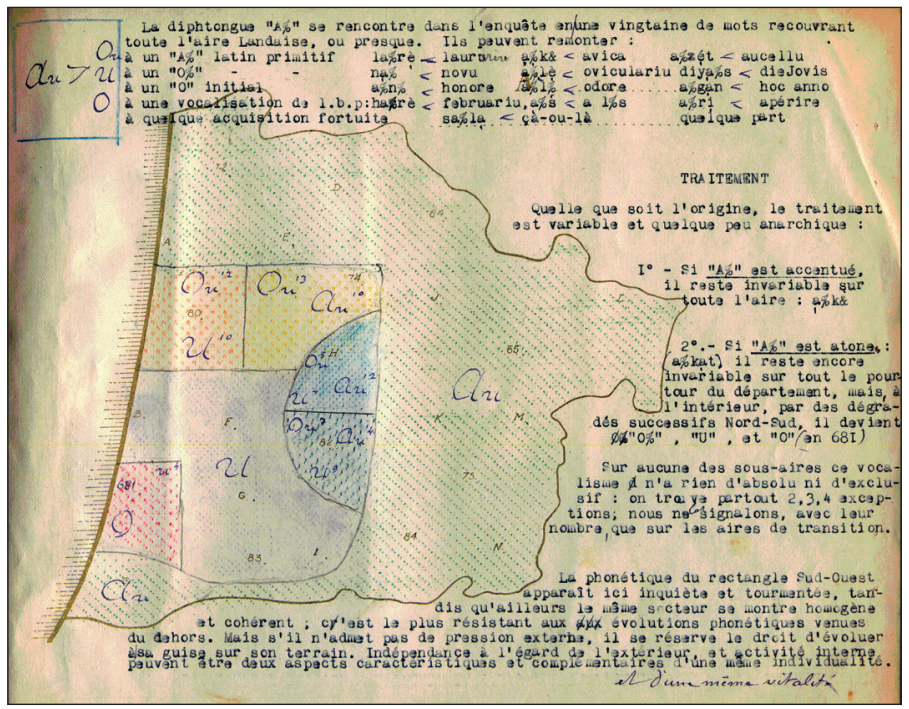

Figure 11. - Carte synthétique :gradient_/aw/.

\section{Des cartes de Lalanne aux sous-ensembles flous : vers une organisation des petits mondes fractals}

Pour résoudre les apories de Lalanne, tous en restant fidèles à la réalité des distributions visualisées, il manquait à tous les prédécesseurs l'outillage nécessaire pour analyser et classifier les tables de données synthétiques dressées par Jean Séguy dans l'ALG 6. Lalanne comme Séguy savaient que Bordeaux était l'œil de la Saintonge, comme Agen, l'œil de la Guyenne; et que sur les pourtours du gascon septentrional s'affrontaient non seulement les caractéristiques de l'occitan périgourdin, de l'occitan de la Charente maritime et de la Charente limousine, mais aussi des parlers d'oïl, le tout formant ce groupe interférentiel donc fractal et flou, ce que Lalanne appelait gavacheries.

La cartographie de sous-ensembles gradients inventée par J. Séguy confortait et démontrait la validité de cette hypothèse des ordres gradués que les mathématiciens, physiciens et naturalistes caractérisent de fractals ou flous.

Mais il y a plus : ce qui était attendu sur les pourtours, se reproduisant, comme naturellement non seulement aux extrêmes, mais partout, aux portes de chaque sous-ensemble territorial défini comme groupe social, et peutêtre comme groupe au sens mathématique du terme, en rapport avec ce 
qui est appelé, en théorie mathématique depuis Zadeh sous-ensemble flou, autrement dit graduel.

Il fallait donc commencer par opérer sur les tables de variables une classification classique, fondée sur une approche binariste d'appartenance à des classes hiérarchiques en introduisant, à la seconde étape du flou dans la classification hiérarchique, en visualisent les faits de classes empiétantes ou floues, que nous appellerons classes culturelles fractales.

Tous les dialectologues du monde ont nécessairement été confrontés à ces faits d'empiètement, en cours de recueil de données de terrain : là où le père disait $X$, la fille, la mère, le gendre ou le voisin disait X' ou X'".

La classification hiérarchique qui ne permet pas de visualiser ces faits d'empiètement est par conséquent prise ici en défaut. Depuis il a été établi que ce type d'organisation ne représentait en aucun cas une caractéristique spécifique du gascon mais une organisation universelle, qui rend compte aussi bien des gradients d'occitanité que de catalanité, de lusitanité, de basquité et de francité en domaine d'oïl.

\section{Série cartographique de gradience et covariance}

\subsection{Visualisation des produits de la diphtongaison conditionnée en contexte droit de coda palatale : œil}

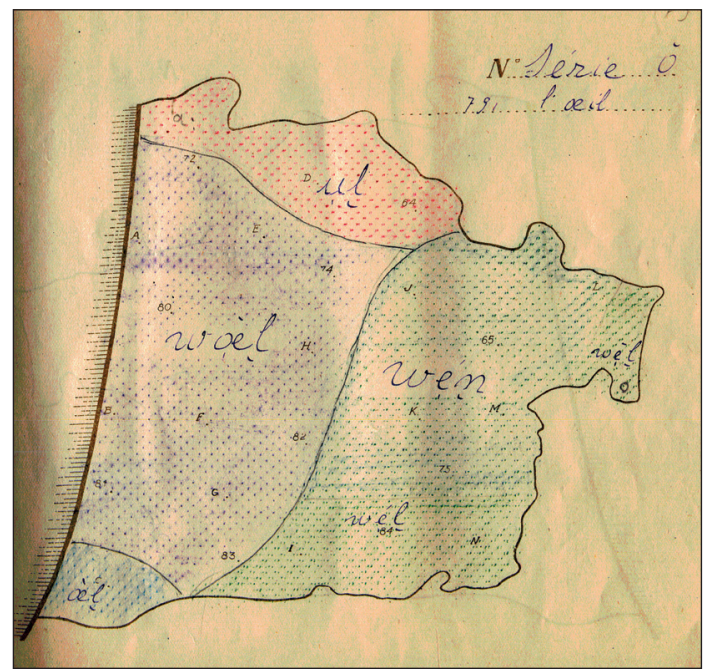

Figure 12. - Carte synthétique : diphtongaison conditionnée /œil/.

La question qui se pose alors n'est pas tant celle des formes de la variabilité, dont la représentation est fonction des modes de calcul des distances 
floues, tordues, graduées, mais de la fonction/des fonctions de la diversité culturelle/langagière, pendant de la diversification observée dans l'ordre du naturel.

\subsection{Série cartographique synthétique des produits de la diphtongai- son conditionnée, en contexte droit palatale en coda : loin}

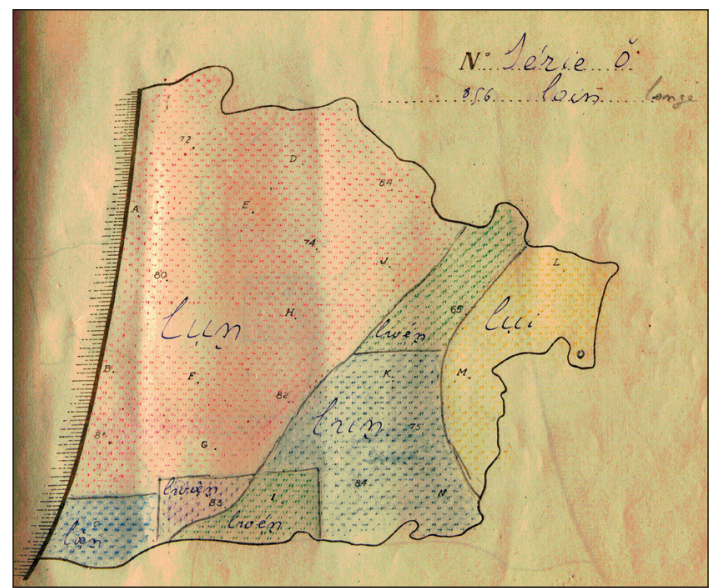

Figure 13. - Carte synthétique : Ioin gradient_labialité/palatalité/covariance.

Et ceci nous conduit tout droit en direction de l'analyse des conditionnements historiques et sociaux depuis le $\mathrm{XII}^{\mathrm{e}}$ siècle, d'une langue à statut dialectal de type occitan aquitanique, que les linguistes ont appelé tour à tour gascon, béarnais.

\section{Conclusion}

Les groupes sociaux restreints ont joué un rôle fondamental, non seulement dans la transmission de cette langue sous des formes fractales, mais dans la formation même de ces objets fractals, que l'analyse géolinguistique représente en sous-ensembles flous qui sont irrémédiablement étrangers aux circonscriptions géopolitiques.

La validation des hypothèses et résultats de Lalanne (1950) a démontré que les arbres de symétrie des divers paramètres obéissent à un partitionnement flou d'objets fractals qui sont loin d'être symétriques.

Le travail qui reste à organiser pour valider ces propositions est immense, mais passionnant : ce chemin ardu est fortement déconseillé aux linguistes seuls, car il constitue un champ d'exploration passionnant pour les chercheurs qui savent, veulent et peuvent travailler en mode trans-échelles. 


\section{RÉFÉRENCES BIBLIOGRAPHIQUES}

Lalanne Théobald, 1949, L'indépendance des aires linguistiques en Gascogne maritime, 2 fascicules ronéotypés, Toulouse.

Lalanne Théobald, 1950, Carnets d'enquête, dans Fonds Séguy, sur le site $<$ occiton.free.fr>.

LaLANnE Théobald, 1950, Cartes analytiques et synthétiques, maquettes sur le site <occiton.free.fr>.

SÉGUY Jean, 1973, Atlas linguistique de la Gascogne (ALG), 6 vol., Paris, Éditions du CNRS.

Fossat Jean-Louis, 1977, «Vers un traitement automatique des données dialectologiques, en dialectométrie », Germanistische Linguistik, n 3-4, p. 311-334.

Fossat Jean-Louis \& Beauchemin Normand, 1978, Cartographie statistique de synthèse des tables de distance de l'ALG, 4 fasc., Toulouse, Unité de service informatique.

FossAT Jean-Louis, 1979, «La théorie de la variation à la lumière des mesures dialectométriques de l'Atlas linguistique et ethnographique de la Gascogne», dans Cahiers de linguistique, d'orientalisme et de slavistique, p. 51-64.

Fossat Jean-Louis et al., 1980, Cartographie des groupes et classes du gascon d'après les tables d'ALG 6 (phonétique, phonologie, verbe, morphosyntaxe), Toulouse, Unité de service informatique. Voir aussi sur <occiton.free.fr>.

Fossat Jean-Louis, 1991, Cartographie de classes de l'Atlas linguistique de la Gascogne, Toulouse, dans Rapport de fin de programme du Conseil régional de Région Midi-Pyrénées «Atlas dialectométrique de la Gascogne», Toulouse, Unité de service informatique.

Fossat Jean-Louis \& SAndouk Zouheir, 1991, Cartographie des groupes, sousgroupes et classes hiérarchiques de l'Atlas linguistique de la Gascogne (phonétique, phonologie, verbe, morphosyntaxe), dans Rapport de fin de programme du Conseil régional de Région Midi-Pyrénées «Atlas dialectométrique de la Gascogne», Toulouse, Unité de service informatique.

Fossat Jean-Louis \& Casanova Hervé, 1996, Cartes distributionnelles du gascon, à partir des tables ALG 6, répertoire Iconographie, 4 fasc., Toulouse, Université Toulouse 2 - Le Mirail.

Fossat Jean-Louis, 2000, «La Chalosse linguistique et ses marqueurs», dans M. Papy et C. Thibon (éds), Chalosse : l'esprit des lieux entre mémoires et histoires, Orthez, p. 291-337.

Fossat Jean-Louis, 2012, «Des données de terrain à leur traitement, 50 ans de recherche », dans Mémoires du terrain, enquêtes, matériaux, traitement des données, Université Lyon 3, p. 189-239.

Fossat Jean-Louis \& ZAFAR Choeb, (à paraître), Méthodes de cartographie de sousensembles dialectaux graduels, Toulouse, Université Toulouse 2 - Le Mirail.

Philps Dennis, 1950, Atlas dialectométrique des Pyrénées centrales, Toulouse, Université Toulouse 2 - Le Mirail.

Philps Dennis, 1985, Atlas dialectométrique des Pyrénées centrales, 2 vol., Toulouse, Université Toulouse 2 - Le Mirail. 